Cahiers $d u$ MONDE RUSSE

\section{Cahiers du monde russe}

Russie - Empire russe - Union soviétique et États indépendants

\title{
Alan Barenberg, Gulag Town, Company Town, Forced Labor and its Legacy in Vorkuta
}

Jean-Paul Depretto

\section{OpenEdition}

Journals

Édition électronique

URL : http://journals.openedition.org/monderusse/8262

DOI : $10.4000 /$ monderusse. 8262

ISSN : $1777-5388$

Éditeur

Éditions de l'EHESS

\section{Édition imprimée}

Date de publication : 1 octobre 2015

Pagination : 868-871

ISBN : 978-2-7132-2507-9

ISSN : $1252-6576$

\section{Référence électronique}

Jean-Paul Depretto, « Alan Barenberg, Gulag Town, Company Town, Forced Labor and its Legacy in Vorkuta », Cahiers du monde russe [En ligne], 56/4 | 2015, mis en ligne le 16 mars 2016, Consulté le 24 septembre 2020. URL : http://journals.openedition.org/monderusse/8262 ; DOI : https://doi.org/ $10.4000 /$ monderusse.8262

Ce document a été généré automatiquement le 24 septembre 2020. 


\title{
Alan Barenberg, Gulag Town, Company Town, Forced Labor and its Legacy in Vorkuta
}

\author{
Jean-Paul Depretto
}

\section{RÉFÉRENCE}

Alan BARENBERG, Gulag Town, Company Town, Forced Labor and its Legacy in Vorkuta, New Haven - Londres : Yale University Press, 2014, 352 p.

Ce livre est issu d'une thèse préparée sous la direction de S. Fitzpatrick. Dès l'introduction, A. Barenberg expose son objectif : « libérer le Goulag de "l'archipel » métaphorique de Solženicyn » (p.14), c'est-à-dire cesser de le considérer comme un autre monde, séparé de la société soviétique. Pour cela, l'auteur a adopté une perspective « au ras du sol » : il s'est concentré sur un cas local, celui de Vorkuta, qui permet de discerner des phénomènes facilement négligés dans les études de niveau national ou régional. Il a mobilisé les archives centrales, régionales et locales de l'état soviétique et du PC, les fonds du musée de Vorkuta, le journal local ainsi que des Mémoires ; il a également mené des interviews de témoins.

2 Le camp de Vorkuta a été créé au début des années trente ; à la fin de cette décennie, il était devenu l'un des complexes concentrationnaires d'URSS ayant la croissance la plus rapide et la mortalité la plus élevée. À côté du camp est née une ville du Goulag qui, dans les années 1950, a commencé à se transformer en ville-usine (company town) par suite de la libération des détenus : le trust d'extraction du charbon a dominé la ville jusque dans les années 1990. L'héritage du travail forcé a pesé lourdement sur l'évolution de Vorkuta longtemps après 1953. Il s'agit ici d'écrire une histoire économique et sociale de Vorkuta des années 1930 au xxi siècle, en examinant les expériences de divers groupes, souvent évoquées à travers des itinéraires individuels. 
3 Le premier chapitre est consacré à la création du complexe de camps. Les effectifs de détenus n'ont pas connu de croissance soutenue avant 1936 : en 1935, ils se situaient aux environs de 3000 personnes. Cependant, la population du camp a connu un accroissement considérable en 1936-1939, atteignant début 1940 le chiffre de 16509 prisonniers. L'année 1936 a été marquée par l'arrivée massive de " contre-révolutionnaires " après le meurtre de Kirov : des centaines de trotskystes ont été transférés à Vorkuta. Cette présence massive des " contre-révolutionnaires " est restée un trait durable de ce camp. Ces prisonniers ont demandé que l'administration reconnaisse leur statut de " politiques » et leur accorde un régime spécial, mais elle s'y est refusée catégoriquement. La grève de la faim a duré presque quatre mois (octobre 1936-février 1937) : le nombre de victimes est inconnu. En deux jours de mars 1938, 524 détenus ont été exécutés, dont de nombreux trotskystes.

4 Les lignes séparant les catégories de statut étaient fluides et mal définies ; un petit nombre de spécialistes et de "colons spéciaux » bénéficiaient de privilèges. Début 1942, environ un tiers des prisonniers étaient autorisés à se déplacer sans escorte ou à vivre en dehors de la « zone ». C'est précisément la crainte de perdre leurs privilèges qui explique en partie la révolte des administrateurs d'une section éloignée du camp, le 24 janvier 1942, à laquelle ont participé à parts égales des " politiques » et des prisonniers de droit commun. Il y eut cinquante condamnations à mort.

5 La guerre s'est traduite par une forte hausse de la mortalité à partir de fin 1942. À ce moment-là, le charbon extrait par les détenus de Vorkuta était devenu d'une importance stratégique, à cause de l'occupation du Donbass par les Allemands.

6 Le deuxième chapitre examine Vorkuta de 1943 à 1947 : le camp a reçu dans cette période des dizaines de milliers de personnes, dont la survie était de plus en plus difficile. Beaucoup d'entre eux étaient d'anciens prisonniers de guerre, des civils suspectés de collaboration ou des rebelles accusés de résistance au pouvoir soviétique. La plupart n'étaient pas russes et la majorité venait des régions occidentales de l'URSS : condamnés pour des raisons politiques, ils purgeaient de longues peines.

7 À partir de 1943, Vorkuta a joué un rôle clef dans l'approvisionnement en charbon de Leningrad. La même année, a été créée officiellement la ville de Vorkuta, séparée du camp : elle avait alors 5500 habitants ( 35000 en 1947). Le directeur du camp, M.N. Malcev, était en même temps chef du trust minier ; dans la plupart des domaines, il ne dépendait que de ses supérieurs du NKVD ; il exerçait tant de pouvoirs que l'autorité du Comité exécutif de la ville était restreinte. Vorkuta n'a eu un gouvernement normal qu'à partir du début des années 1950. L'auteur insiste sur la fluidité des identités : les familles mixtes, composées de détenus et de non-prisonniers, n'avaient pas de place claire dans la hiérarchie sociale.

8 Au total, la guerre occupe une place spéciale dans la mythologie de Vorkuta, célébrée pour les sacrifices désintéressés de ses « travailleurs » venus en aide à Leningrad. Mais c'est à ce moment de " gloire " que le taux de mortalité a été le plus élevé de l'histoire du camp.

9 Le troisième chapitre analyse la recherche de la "normalité » sous le stalinisme d'après-guerre (1947-1953). La construction de l'hôpital des enfants, achevée en 1950, représente l'un des symboles de cette "normalité ». Dans les camps, la mortalité a été presque divisée par vingt entre 1943 et 1953. En 1950-1952, les prisonniers ont commencé à recevoir des salaires qui amélioraient leur situation. Le système des 
crédits en jours de travail permettait de raccourcir les peines pour crimes légers. Mais l'année 1948 a vu la création du " camp spécial » visant à isoler et punir les plus dangereux ennemis de l'État : il concentrait les prisonniers politiques appartenant aux nationalités des frontières occidentales de l'URSS (Ukrainiens et Baltes). Ces minorités ont créé des organisations nationalistes clandestines partout présentes à la fin de la période stalinienne.

10 À long terme, des bases ont été posées pour l'expansion de Vorkuta en tant que ville-usine ; elle a été dotée d'institutions nominalement indépendantes du camp. Les anciens prisonniers constituaient la majorité de la considérable hausse de la population ; à partir de 1948, la cité connut un véritable baby-boom. La "normalité " était toutefois précaire : il est pratiquement impossible de séparer la violence arbitraire des camps et le monde civilisé de la ville soviétique.

11 Le quatrième chapitre est intitulé " Vorkuta en crise ». À l'été 1953, la grève de 15000 détenus du « camp spécial » constitue le signe le plus patent de cette crise profonde qui saisit le complexe concentrationnaire après la mort de Stalin. Elle représente un des plus importants actes de résistance massive de l'histoire du Goulag. Des milliers de prisonniers ont obtenu le droit de se déplacer sans garde ou de vivre en dehors du camp. L'amnistie du 27 mars 1953, qui au total a libéré plus d'un million de prisonniers, a été restreinte à Vorkuta en raison du pourcentage élevé de " contre-révolutionnaires "; elle n'en a pas moins joué un rôle décisif dans la genèse de la grève. Les administrations des mines et des camps étaient presque paralysées à l'été 1953 : une " guerre de territoire " opposait le ministère des Charbonnages à ceux de l'Intérieur et de la Justice pour le contrôle de la main-d'œuvre.

12 La résistance des détenus du " camp spécial » a été favorisée par le fait que ce dernier enfermait, outre d'anciens rebelles nationalistes, des vétérans de l'Armée rouge. La grève exploitait la crise d'autorité à Vorkuta ; grévistes, administration du camp et fonctionnaires de la mine ont coopéré durant tout le conflit. Ce dernier a été bien coordonné par les organisations nationalistes; il s'est terminé tragiquement (53 morts). En 1954, les « camps spéciaux » furent supprimés.

13 En 1956-1957, la "zone » se rétrécissait : le fil barbelé reculait et une partie de l'espace du camp était intégrée dans la ville-usine. Pour obtenir une main-d'œuvre qualifiée et fiable, on a converti les " esclaves " des camps en "serfs » liés à leur entreprise. En août 1955, le Conseil des ministres a décidé que seuls des non-prisonniers travailleraient au fond ${ }^{1}$. Désormais, il s'agissait de trouver des dizaines de milliers d'ouvriers pour remplacer les détenus.

14 Le cinquième chapitre aborde la transformation de la ville du Goulag en ville-usine. Environ 105000 prisonniers ont été libérés entre 1953 et 1958 ; il s'agissait maintenant de trouver des spécialistes et des ouvriers pour remplacer plus de 20000 détenus employés dans les mines (presque $70 \%$ de la main-d'œuvre), ainsi que des milliers d'exilés amnistiés. Le trust minier a d'abord compté sur l'orgnabor (1954-1955) ; en 1955-1960, plus de 15000 anciens soldats ont été recrutés pour Vorkuta. Beaucoup de jeunes volontaires ont été envoyés par le Komsomol en république Komi. Des efforts concertés ont été menés pour recruter du personnel diplômé d'instituts ou d'écoles techniques. En 1960, presque aucune femme ne travaillait plus au fond ; les femmes de Vorkuta ont cherché en vain à obtenir le droit de descendre à nouveau dans la mine.

À partir du milieu des années 1950, des dizaines de milliers de personnes ont choisi de venir s'installer à Vorkuta : elles fuyaient la pauvreté et étaient attirées par les emplois 
abondants, les occasions de se qualifier, ainsi que les promesses de hauts salaires. Cet attrait pour le « long rouble » n'excluait pas d'autres motivations, comme la fierté de participer à la construction d'une ville nouvelle.

Les autorités locales ont remporté des succès dans le domaine de la formation, qui était un facteur très important d'intégration. Mais la pénurie chronique en matière de logement explique les fréquents départs des migrants. Le turnover était élevé dans les mines et plus encore dans la construction, mais moins que dans les années 1930.

17 Au total, au début des années 1960, les autorités locales pouvaient se vanter de réussites significatives dans l'intégration des nouvelles recrues.

Le sixième chapitre est intitulé " Des prisonniers aux citoyens ? ». Avec leur famille, les ex-prisonniers constituaient un tiers de la population de Vorkuta à la fin des années 1950. Ils rencontraient des obstacles : la discrimination légale, la surveillance policière, la méfiance des voisins. Le KGB suivait de près trois types d'opposition potentielle : politique, nationaliste et religieuse, en particulier les témoins de Jéhovah. Les archives $\mathrm{du}$ trust minier ont permis à $\mathrm{A}$. Barenberg de mener une remarquable étude de la discrimination en matière d'emploi des spécialistes : ses conclusions sont nuancées. Les anciens prisonniers ont certes été écartés des postes les plus élevés, mais il n'existait pas d'interdiction explicite de les embaucher. La discrimination officielle était sapée par la pénurie de main-d'œuvre et le turnover.

Des dizaines de milliers de ces anciens détenus ont pu bâtir des vies normales à Vorkuta et réintégrer la société soviétique, qui a su les accueillir.

20 L'épilogue conduit le récit jusqu'à nos jours, trop brièvement à mon goût. La grève des mineurs de 1989 est rapidement évoquée. L'effondrement des années 1990 est bien décrit. En 2003, les mines de Vorkuta ont été achetées par le conglomérat Severstal', ce qui augure peut-être d'un meilleur avenir. Le livre se termine par une analyse du traitement local de la mémoire du Goulag.

21 Cet excellent travail montre une fois de plus ce que peuvent apporter à la compréhension de l'expérience soviétique des monographies locales bien conçues. On regrettera seulement l'absence de bibliographie et le caractère minimaliste des indications portant sur les archives (p. 279-280).

\section{NOTES}

1. Mais le secteur de la construction a continué à employer des détenus jusque dans les années 1980. 


\section{AUTEURS}

JEAN-PAUL DEPRETTO

Université Toulouse-Le Mirail - Cercec, Ehess 